\title{
GANGOTRI mission concept on the glacial key to the Amazonian climate of Mars
}

\section{Coordinating authors:}

Suniti Karunatillake (legal last name: Walimuni

Devage), Louisiana State University ${ }^{1}$

Ali Bramson, Purdue University

Kris Zacny, Honeybee Robotics

Colin Dundas, U.S. Geological Survey

Lujendra Ojha, Rutgers University

Corresponding author email address: sunitiw@lsu.edu

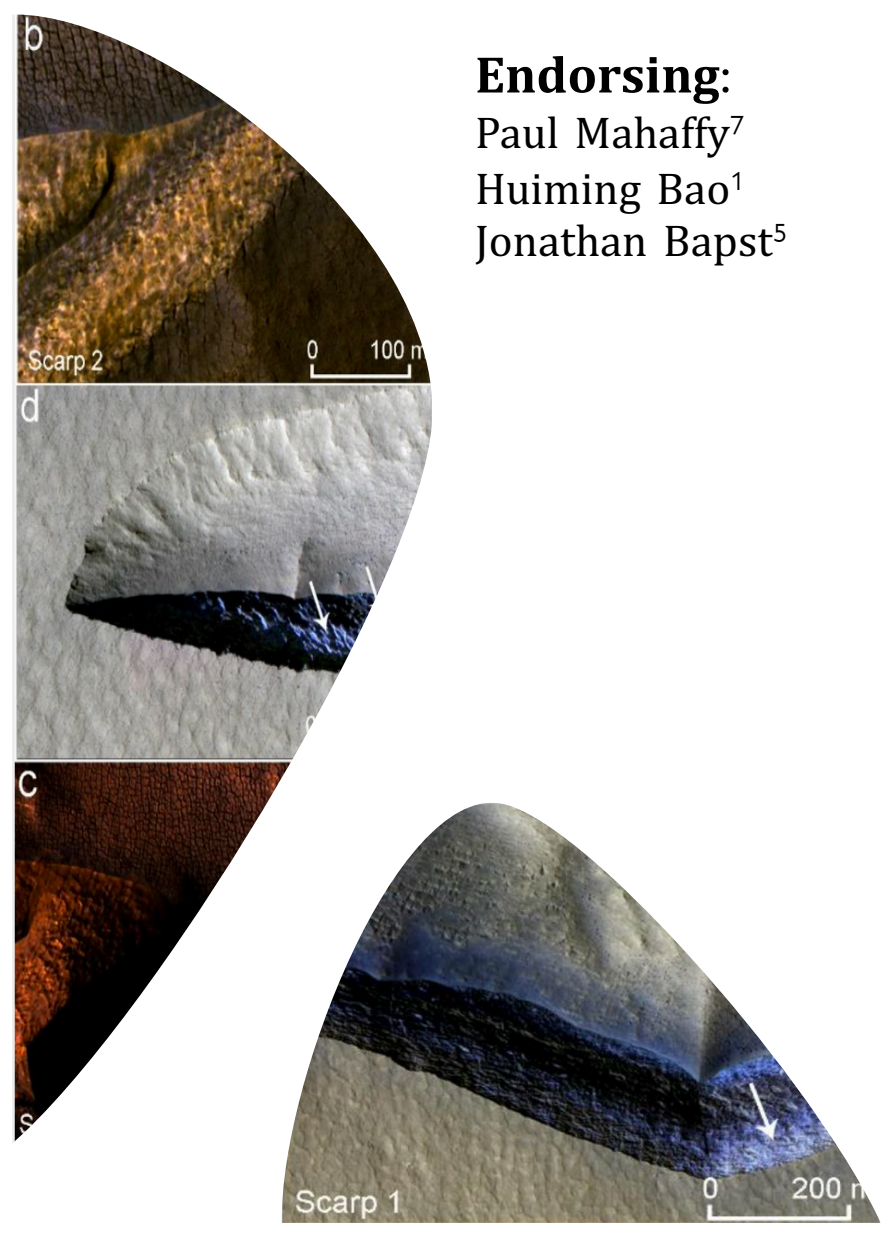

Technology development authors:

Juan Lorenzo ${ }^{1}$

Katherine Mesick, Los Alamos National Laboratory ${ }^{6}$ Heather Franz, Goddard Space Flight Center ${ }^{7}$ Jose Rodriguez-Manfredi, Centro de Astrobiologia INTA-CSIC, Spain

Daniel Coupland 6

Nathan Bramall, Leiden Measurement Technology Peter Bertone ${ }^{3}$

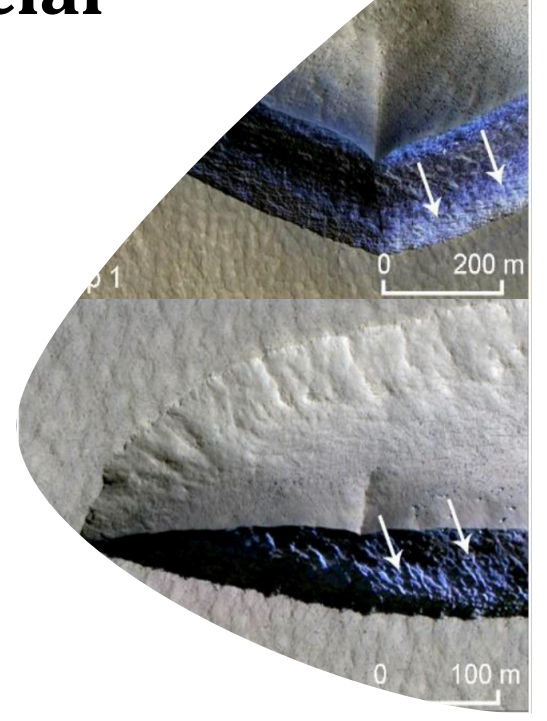

Science authors:

Oded Aharonson, Planetary Science Institute Eran Vos, Weizmann institute of science, Israel Donald R. Hood, Texas A\&M Deanne Rogers, Stony Brook University Joseph Levy, Colgate University Peter Doran ${ }^{1}$ Kathleen Mandt, JHU APL ${ }^{2}$ Jack Wilson ${ }^{2}$

Emily B. Hughes ${ }^{1}$, Wesleyan University Heidi Fuqua-Haviland, NASA-Marshall Space Flight Center ${ }^{3}$ Jeff Moersch, University of Tennessee, Knoxville Scott M. Perl, Jet Propulsion Laboratory 5 Dewan Mohammad Enamul Haque ${ }^{1}$, University of Dhaka J.R. Skok, SETI Institute Harish, Physical Research Laboratory, India ${ }^{4}$ Vijayan S. ${ }^{4}$ Anil Bhardwaj ${ }^{4}$ Brent Christner, University of Florida Hanna Sizemore, Planetary Science Institute Akos Kereszturi, Research Centre for Astronomy and Earth Sciences, Hungary Norbert Schorghofer, Planetary Science Institute Kurt Retherford, South West Research Institute Paul Niles, Johnson Space Center 


\section{GANGOTRI mission concept}

Motivation and overview. The wealth of geologic information bound in Martian ice, including climate cycles, potential biomarkers, atmospheric particulates, and sources of $\mathrm{H}_{2} \mathrm{O}$ that may drive alteration within the critical zone $(\mathrm{CZ}$ : zone of interaction between the atmosphere and the porous upper crust) has long been recognized. Much progress has been made in the last decade to address the ice, habitability, and CZ themes of the NASA Visions and Voyages 2019-2022 Decadal Survey (DS) [NRC, 2011], mid-term review (MTR) [NAS, 2018], and NASA's science plan (SP) [2014]. Those include: polar ice sheets [e.g., Bapst et al., 2018, 2019; Becerra et al., 2017; Bramson et al., 2019; Campbell and Morgan, 2018; Grima et al., 2009; Landis et al., 2016; Ojha et al., 2019; Orosei et al., 2018; Phillips et al., 2011; Putzig et al., 2018; Smith and Holt, 2010; Smith et al., 2013, 2016; Sori and Bramson, 2019; Whitten et al., 2017] and midlatitudinal ice [e.g., Adeli et al., 2019; Baker and Carter, 2019a,b; Bramson et al., 2015, 2017; ; Brough et al., 2019; Byrne et al., 2009; Dickson et al., 2012; Dundas et al., 2014, 2018; Holt et al., 2008; Kadish and Head, 2011; Lefort et al., 2009; Orgel et al., 2019; Pathare et al., 2018; Petersen et al., 2018; ; Piqueux et al., 2019; Plaut et al., 2009; Ramsdale et al., 2019; Séjourné et al., 2019; Sizemore et al., 2015; Stuurman et al., 2016; Viola et al., 2015]. Despite the advances, many outstanding questions remain, and new ones have emerged; many have been pushed even further to the forefront of exploration [cf. Bramson et al., 2020]. Ice in the midlatitudes is a key in situ resource for future human exploration and contains a wealth of information regarding the climatic history of Mars. Recent midlatitudinal Martian ice discoveries by Dundas et al. [2018] and Harish et al. [2020], unknown during the DS polar mission studies [cf. Appendices D, G of NRC, 2011], provide a notable opportunity to explore the evolution of ice beyond the poles. Likewise, MTR emphasizes them as key to future exploration [NAS, 2018]. Remote sensing suggests compaction resembling terrestrial glaciers, but with limited evidence for flow. The glaciers are ablating as evident from both scarp activity and collapsed boulder fields [Dundas et al., 2018]. Accordingly, our Glacial Ares Nimbus \& Geologic Origins Thermal Riddling Investigation (GANGOTRI) mission concept - aptly eponymous with the large and fast-receding Himalayan glacier, Gangotri-would investigate the geologic origins of midlatitudinal Martian glaciers. GANGOTRI would enable key insight into Amazonian climate evolution, especially precipitation events (i.e., nimbus for short), via a hybrid thermo-mechanical drill called RedWater [Zacny et al. 2019, 2020] for deep and possibly multiple englacial sampling. GANGOTRI would use regolith compositional measurements to characterize iceregolith interaction, and stable isotope measurements to characterize fundamental exchange processes of major ice reservoirs. Meanwhile, geophysical observations would cross-calibrate composition with some sensitivity to the presence of brines or meltwater.

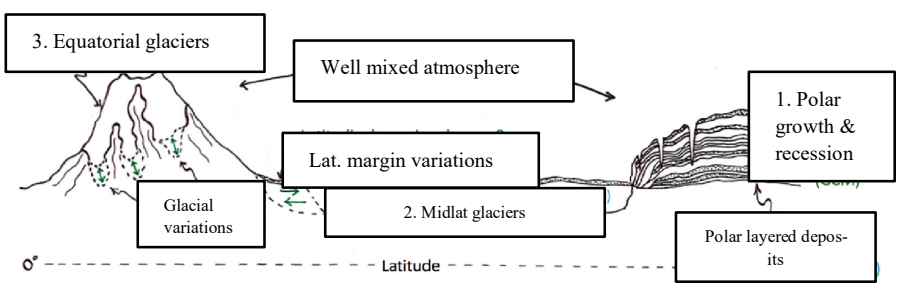

Fig 1. Schematic of the latest $\mathrm{H}_{2} \mathrm{O}$ reservoir model for Mars by Vos et al. [2019], testable by characterizing deuterium to hydrogen $(\mathrm{D} / \mathrm{H})$ isotopic ratio in midlatitudinal glaciers (labeled the $2^{\text {nd }}$ of the three reservoirs). While uncommon, possible layering within midlatitudinal glaciers [Dundas et al., 2018] increases the likelihood of an isotopically variable counterpart to Reservoir 1.

\section{Objectives and Rationale}

Science Objectives. As enumerated in the Science Traceability Matrix (STM) (Karunatillake et $a l ., 2020)$ GANGOTRI supports two science goals and one human exploration goal that advance 


\section{GANGOTRI mission concept}

overarching themes of the Committee on Astrobiology and Planetary Sciences (CAPS) report [NAS, 2017b], DS, MTR, and SP. Summary descriptions follow.

Geological Science Goal: understanding climate-driven processes of mid-latitudinal glaciers as a key $\mathrm{H}_{2} \mathrm{O}$ reservoir within Mars's CZ. Mechanistic links among orbital variations, climate, and glacial stratigraphy remain unknown on Mars, due to the lack of correlating analytical methods [cf., Hvidberg et al., 2012]. That can be resolved by isotopically sampling in the top 100 $\mathrm{m}$, especially to reveal climate oscillations unseen in glacial stratigraphy and to probe recent precession-driven climate cycles [Vos et al., 2019]. Isotopic variations can characterize the temporal evolution of $\mathrm{H}_{2} \mathrm{O}$ reservoirs on Mars and underlying climate conditions, as exemplified by the latest triple-reservoir $\mathrm{H}_{2} \mathrm{O}$ exchange model [Vos et al., 2019] shown in Fig 1. The englacial D/H ratio in the midlatitudes, such as at Milankovic crater $\left(54.8^{\circ} \mathrm{N}, 212^{\circ} \mathrm{E}\right)$, would resolve the lack of a point constraint to calibrate and interpret such isotopic models. Conversely, deviations from model predictions would place new constraints on the average $\mathrm{D} / \mathrm{H}$ ratio of the reservoirs, and the temperature differences among the reservoirs at the time of ice accumulation.

The $\mathrm{D} / \mathrm{H}$ profile can also resolve inconsistencies in global $\mathrm{H}_{2} \mathrm{O}$ isotopic models. For example, spectroscopic data of the current Martian atmosphere from Earth-based telescopes indicate that the $\mathrm{D} / \mathrm{H}$ ratio varies greatly in space and time with a range from 1-10 times of that of terrestrial water (e.g., Vienna Standard Mean Ocean Water, VSMOW), apparently contradicting the predicted range of 1 to 2 times that of VSMOW [cf., Good et al., 2015] by the global water isotope model for Mars [Krasnopolsky, 2015]. The large, observed D/H ratio variation may indicate still unknown effects of low temperature on isotopic diffusion in $\mathrm{H}_{2} \mathrm{O}$ ices [cf. Livingston et al., 2002; Markland et al., 2008]. Consequently, if D/H ratio variations in Martian ice profiles are several multiples of VSMOW, low-temperature anomalies would be confirmed as an essential reference for low temperature ice diffusion experiments and theoretical modeling for planetary settings. Alternatively, if the $\mathrm{D} / \mathrm{H}$ profile resembles terrestrial ice, the significance of temperature-driven $\mathrm{D} / \mathrm{H}$ fractionations would diminish for ice evolution in solar system bodies.

GANGOTRI would also examine potential geochemical signatures of climate evolution, using a mid-latitudinal glacier as an Amazonian case study. For example, Niles and Michalski [2009] hypothesized that siliciclastics undergo low-pH alteration englacially by thin films of water through radiant heating, facilitating reactions between atmospherically sourced dust and sulfate-rich aerosols. As a temperature proxy, D/H variability would further support our geochemical work on testing such hypotheses.

Biological Science Goal: determine the habitability and biomarker preservation potential of young Martian glaciations. The distribution of organics within ice on Mars is unknown. Presumed isochrons discovered in the martian polar layered deposits [e.g., Ojha et al., 2019; Smith et al., 2016, 2020] suggest that ice on Mars may preserve past time horizons as it does on Earth. As Mars has planet-encircling dust storms, climate-driven aeolian processes that could transport dust, ash, and organics are plausible. Consequently, we would examine whether organics and microorganisms have been deposited, immured, or preserved at some level in midlatitudinal ice deposits in association with aeolian-deposited englacial dust, including as a point reference for the global abundance of atmospheric organics during the Amazonian. Non-invasive detection of brines would further clarify the habitability potential.

Human Exploration Goal: understanding the utility of glacial ice as an in situ resource

(ISR). The reconnaissance of glacial morphology and characterization of depth to ground ice would result in the first ground-truth reference volume of glacial ice as a possible ISR. The general constraints on the relative purity of the ice can help identify the optimal $\mathrm{H}_{2} \mathrm{O}$ extraction and 


\section{GANGOTRI mission concept}

purification methods [cf., Zacny et al., 2012]. Lastly, work on our biological goal will help set thresholds for forward and backward contamination, enabling technological developments on using readily accessible Martian ice as a key ISR by future human explorers.

Science Rationale. STM (Karunatillake et al., 2020) explicitly ties NASA goals (i.e., CAPS report, DS, MTR, and SP), mission goals, science objectives (i.e., aims), payload design, and science-mission formation.

Technology Rationale. Our proposed mission supports future technology needs motivated by MTR. Examples include the development of a drill and integrated biomarker and siliciclastic characterization instruments, in turn providing the technology readiness needed for future applications, like sampling the subglacial environments on Europa [cf. NAS, 2018].

Mission Concept Summary. While not funded, reviewers of our GANGOTRI mission concept proposed to the 2023-2032 decadal mission concept study solicitation highlighted the potential for Flagship class and the importance of maturing the drill design, thus here we include it only to establish a reference in relation to STM (Karunatillake et al. 2020). Assessing the tradespace of what may be achievable at lower cost levels is desirable and we present a preliminary instrument tradespace to lower costs without undermining the science and human exploration goals enumerated in STM. We have also advanced the drill concept with the RedWater drill design, relying on research by Honeybee Robotics (use of firm names for descriptive purposes only, without implying endorsement by the U.S. Government).

GANGOTRI Spacecraft (Spacecraft Architecture, Table SA (Karunatillake et al., 2020) As on MSL [Grotzinger et al., 2012], communication with Earth via the deep space network and Mars orbiters would use a fixed low-gain antenna and a pointable high-gain antenna.

Entry, Descent and Landing (EDL) Platform would use the MSL-M2020 Entry, Descent and Landing Instrumentation 2 (MEDLI2) suite that would enable Range Trigger and Terrain Relative Navigation (TRN) to target landing within $10 \mathrm{~km}$ of the target along with sky crane delivery [e.g., Abilleira et al., 2019], considerably smaller than MSL's 19x7 km landing ellipse [Martin et al., 2015].

Rover design modifies MSL-M2020 while retaining Radioisotope Power Systems (RPS). As described subsequently, it would contain four key instruments and an arm with a deep drill and three additional drill-mounted instruments.

The Rotorcraft would use a Mars Helicopter Scout (MHS) launching within $100 \mathrm{~m}$ of the rover [e.g., Agle \& Johnson, 2019; Balaram et al., 2018], enabling aerial surveys of glacial scarp fronts, ravines, and other instabilities, as landscape features never examined by prior in situ missions (e.g., including miniature synthetic aperture radar for shallow subsurface surveys, if the technology matures). Additionally, the MHS adaptation would map geology to establish broader context using a Mars2020 heritage nadir optical mapping camera and a stereoscopic pair for navigation and scouting [Balaram et al., 2018].

Summary Science Payload

The Entry, Descent and Landing (EDL) Platform would have an EDL camera and radar altimeter system to characterize the terrain pre-landing.

The Rover provides a mobile-to-stationary platform for the payload to identify the optimal drill site and develop environmental and geologic context. Mobility ensures enhanced insight into lateral variations in fundamental properties of the ice sheet, especially stable isotopes.

The internal deck-mounted Sample Analysis at Mars (SAM) [Mahaffy et al., 2012] would primarily sample variations in the $\mathrm{D} / \mathrm{H}$ ratio of water in glacial ice delivered from drilling, along with opportunistic measurements of atmospheric $\mathrm{D} / \mathrm{H}$ for reference. With six gas chromatograph 


\section{GANGOTRI mission concept}

columns, and the capability to use these in tandem with a mass spectrometer for gas chromatograph mass spectroscopy (GCMS), the SAM instrument suite can search for organic compounds, complementing the drill-mounted payload [e.g., Franz et al., 2014, 2017; Mahaffy et al., 2015]. The arm-mounted Gamma-Ray and Neutron Spectrometer (GRNS) subsystem would provide rapid detection of water ice and bulk geochemistry within a meter of the surface to identify target drilling sites. The GRNS is baselined as one module of the EPICS instrument [Mesick et al., 2018], a low-resource combined GRNS that incorporates elpasolite scintillator material and silicon photomultipliers (SiPMs) (Fig 2). Notably, SiPMs require only tens of volts bias, simplifying the design and reducing the possibility of high-voltage breakdowns in landed environments.

The wheel-mounted acoustic seismometer array consists of low-mass ( $9 \mathrm{~g}$ each) piezo-ceramic seismic sensors and pulsers integrated into the rover wheels to ensure best ground coupling. Piezo-ceramics can also generate controlled low-energy signals (only micrometers displacement). Over a rover transect, active pulsing measurements can provide continuous subsurface maps ( $\sim$ meter depth) of mechanical properties to predict and confirm observations from other payload instruments, particularly GRNS, searching for buried ice as well as the presence of brines or fluids [Lorenzo et al., 2019].

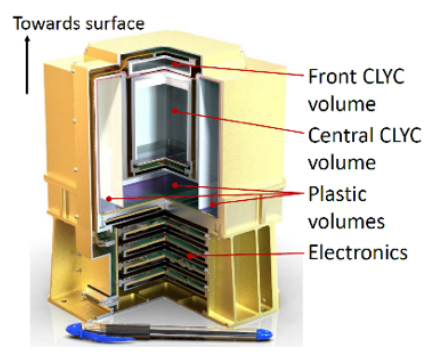

Fig 2. Rendering of the integrated EPICS GRNS with cross section to show detection volumes. Neutrons are detected via ${ }^{6} \mathrm{Li}(\mathrm{n}$, alpha)T capture within $\mathrm{Cs}_{2} \mathrm{LiYCl}_{6}$ (CLYC) and separated from gammas with pulseshape discrimination.

Deck-mounted MEDA of M2020 design [Rodriguez-Manfredi et al., 2014]. MEDA sensors would be on the mast, deck, front, and interior of the rover's body, with $\sim 5.5 \mathrm{~kg}$ for all components, consisting of five air temperature sensors; radiation and dust sensor; relative humidity sensor; thermal infrared sensor; wind sensors; instrument controller; and pressure sensor. Rover cameras would use MSL panoramic and navigation camera design [Bell et al., 2017; Malin et al., 2017], along with engineering cameras [Maki et al., 2012]. The mast-mounted camera system would be modified to complement the capabilities of the Mini-TES.

Mast-mounted MiniTES would adapt the MER-MiniTES design [Christensen et al., 2003] or a Raman spectrometer, for the first cross-validation of a glacial regolith composition on Mars.

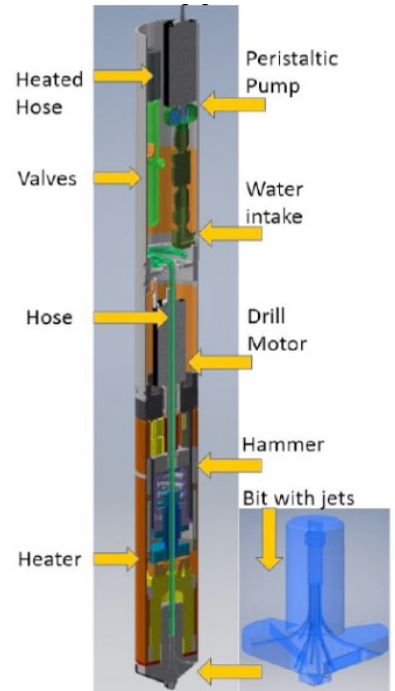

Fig 3. Conceptual illustration of a hybrid thermo-mechanical drill called RedWater, to be scaled down for our mission. Our design would include downhole instruments.

The RedWater Drill (Fig 3) constitutes the core of science operations for englacial characterization, advancing the strategies considered previously by the planetary community [e.g., Beegle et al., 2009; Carsey et al., 2005; Glass et al., 2014; Hecht and Saunders, 2003; McKay et al., 2013; Smith et al., 2017; Stamenković et al., 2019; Zacny et al., 2008, 2019, 2020]. A key development need is incorporating a sample preparation-delivery system to the deck-mounted SAM. RedWater uses traditional coiled tubing drilling approach with a bottom hole assembly consisting of a motor and drill bit, to be augmented with thermal ablation. Compressed Martian atmosphere is used to blow cuttings out of the hole. Currently RedWater is under development for Mars ISRU applications with a goal of reaching 10-30 m through siliciclastic overburden and into ice. Deeper drilling would require longer coiled tubing which adds incremental mass 


\section{GANGOTRI mission concept}

and volume to the system. The same approach is being used to drill on the Moon as part of the LISTER heat flow probe (launch on CLPS lander in 2022). Englacial observations would use the following three drill-mounted instruments.

The Dust Logger uses laser-light scattering to measure englacial variations in dust concentrations with millimeter-scale resolution [cf., Bramall et al., 2005]. It has been successfully deployed in terrestrial ice sheets in Antarctica and Greenland and can survive depths of $4 \mathrm{~km}$ on Earth. It is a few centimeters in diameter and roughly $1 \mathrm{~m}$ long.

The SURFFS UV Fluorometer (Fig 4) can survive $500 \mathrm{~m}+$ terrestrial water deployments. With up to 4 excitation wavelengths and 16 emission channels, it can detected microbial cells down to the glacier's native $50-100$ cells $/ \mathrm{ml}$ in concentration with high depth resolution ( a few $\mathrm{mm})$ in real time during descent. Additional sensitivity enhancements are ongoing. Polycyclic aromatic hydrocarbons are detectable at $\mu \mathrm{g} / \mathrm{kg}$ threshold [cf., Clark et al., 2017].

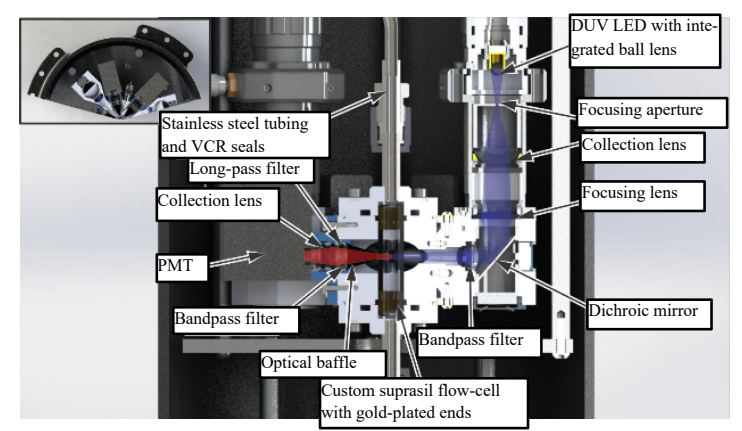

Fig 4. Double-vertical cross-sectional view of the fluorometer. One plane contains the optical axis of the emission system and the other plane contains the optical axis of the excitation system. Light from a DUV LED is collected by a ball lens and focused into the flow cell after passing through an excitation filter. An optical baffle on the emission collection system reduces stray light.

The UV Microscope would serially filter the sample using three different size filters, nominally 10, 1, and $0.1 \mu \mathrm{m}$ [Quinn et al., 2019]. It would image in darkfield in four different wavelengths and excite fluorescence at four different wavelengths, including D-UV wavelengths of 200 to $400 \mathrm{~nm}$, and observe it in five independent emission bands. Samples will have native their fluorescence characterized and can be stained to allow targeted structural studies. The data products are z-stacked images and site-processed metadata. The current resolution is $0.8 \mu \mathrm{m}$ with a $15 \mathrm{x}$ objective and $0.35 \mu \mathrm{m}$ with a $40 \mathrm{x}$ objective.

Instrument tradespace. The drilling depth $(10-30 \mathrm{~m})$ would be optimized as a function of accessing the record of paleoclimate evolution, volumetric constraints on ice for ISR purposes, and mission operation time, with a single Mars year nominal mission expected for maximum insight into the climate over one orbital revolution and into the variations within the glacial column. Alternative configurations for SAM that do not include the complexity of the Gas Chromatograph (GC) system are summarized in SAM Minimal Adaptation, Table SMA, (Karunatillake et al., 2020). Another key tradespace would be single or dual RPS systems. With a single RPS, electrical and thermal power interconnect between the drill and the rover [e.g., Carsey et al., 2005], optimizing energy and mass constraints for the spacecraft. The primary alternatives in that scenario would be between mounting the RPS on the drill (i.e., maximizing and simplifying thermal energy supply for drilling) versus the rover deck (i.e., simplifying electrical supply to the rover). In contrast, a dual RPS system uses a dedicated thermal and electricity source for the drill instrumentation, eliminating the need for thermal and electrical coupling between the drill and rover, and simplifying the drill deployment design. In that case, the drill's RPS would be finless resembling the HOMER-15 concept by Carsey et al. [2005]. A corresponding drill design would be $26-28 \mathrm{~cm}$ in diameter and $\sim 59 \mathrm{~cm}$ long, with the RPS about $49 \mathrm{~kg}$ mass (https://rps.nasa.gov). 


\section{GANGOTRI mission concept}

We identify four levels of tradespace priorities. Level 1 would satisfy the mission goals, while each additional level enhances the degree of interpretation and hypothesis testing (Table STM (Karunatillake et al., 2020)): (1A) drill integrated UV fluorometer for biomarker detection; (1B) drill deployment and sample preparation and delivery system; (1C) SAM minimal adaptation (Table SMA) for englacial D/H isotopic ratio data; (1D) Navigation Cameras, based on M2020 EECAM, to visually identify optimal glacial drilling site; and (1E) MEDA system to provide continuous temperature, pressure, humidity and wind velocity measurements for comprehensive atmospheric process insight over a Martian year for refining current global circulation models on ice stability and exchange with the atmosphere.

Level 2 would enhance site selection and increases englacial isotopic insight: (2A) Arm Imager for high-resolution images of the surface to guide drill deployment; (2B) minimal GRNS module to characterize depth to ground ice; $(2 \mathrm{C})$ mast mounted cameras to map the terrain based on MSL's MastCam; and (2D) SAM without GC (Table SMA (Karunatillake et al., 2020)) for detailed englacial $\mathrm{D} / \mathrm{H}$.

Level 3 would provide contextual composition, maximizes englacial information, and offers complementary borehole characterization: (3A) drill integrated dust logger and microscope to identify englacial dust-ash layers and siliciclastic chemical weathering processes; (3B) full GRNS module to characterize bulk chemistry at decimeter depths; and (3C) MiniTES-ana$\log$ /Raman incorporated into the rover mast for mineralogical differences between dust of global provenance and local soils, along with $\mathrm{D} / \mathrm{H}$ isotopic ratio data (Fig 5), and biomarkers.

Level 4 would provide reconnoitering and environmental characterization: (4A) MHS to identify distally hazardous terrain, map geology with nadir and stereoscopic imaging; and (4B) acoustic seismometer system supplementing GRNS-based characterization of regolith stratification.

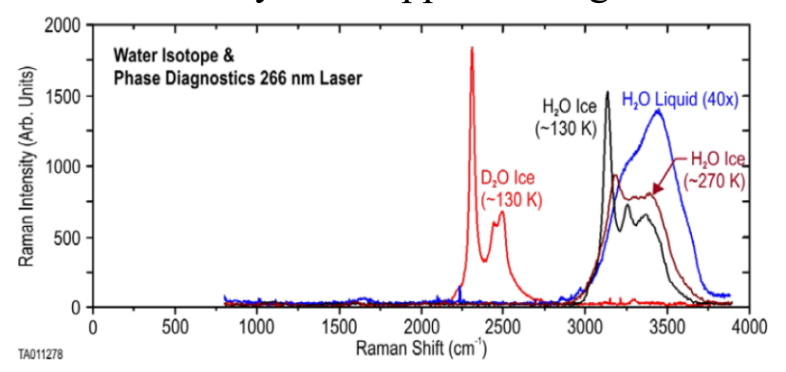

Fig 5. Demonstration of SwRI's TRL 4 cavity breadboard for Raman spectroscopy to estimate $\mathrm{D} / \mathrm{H}$ from distinct spectral peaks for $\mathrm{D} 2 \mathrm{O}$ and H2O for a Deep-UV $266 \mathrm{~nm}$ laser source. Improvements are underway to the optical couplings, detector, and laser sources for enhanced quality [Retherford et al., 2019].

\section{Recommended technological developments for the next decade include:}

1.Thermo-mechanical drilling in englacial heterogeneities (e.g., siliciclastic lags), and how the drill's thermal flux may cause isotopic fractionation effects in acquired samples.

2. Sample preparation and delivery from borehole to deck-mounted SAM.

3. Integration of fluorometer, dust logger, and microscope with a drill.

4. Piezoelectric acoustic seismometer design and integration in rover wheels.

5. Adapting MHS for geologic mapping and hazard characterization.

Relevance to other target bodies. Intended englacial sampling would also yield tools for interrogating ice-rich environments throughout the solar system, especially of icy bodies like Europa which are considered prime exploratory targets for extant life [NAS, 2018]. In turn, that would curtail risk and cost for future missions to Europa by using Mars as a "stepping stone" towards the Ocean Worlds Exploration Program mandated by the 2016 Congressional Bill.

Planetary Protection Assessment. The mission's glacial target may transform into a special region due to drill induced melting-sublimating processes (Appendix G of DS). Consequently, Planetary Protection Category IVc applies, where mission development can help to advance 


\section{GANGOTRI mission concept}

planetary protection strategies on minimizing forward contamination when future missions begin englacial sampling at possibly inhabited bodies such as Europa. That would also involve modeling the thermal flux and associated melt zone stability as a function of elapsed half-lives of a drill-mounted RPS. Identified biological effects would refine the RPS tradespace.

Cost Assessment (Table CA (Karunatillake et al., 2020)). Mission development would identify how to reduce cost of the most expensive components (e.g., RPS integrated drill, borehole sample preparation and delivery to SAM) within the tradespace priorities. The rough order of magnitude (ROM) calculation is estimated using DS's Appendix G for reference. The minimum ROM cost for the 1 Mars Year baseline (2 Earth years) is $\sim \$ 940 \mathrm{M}$ (FY19, Table CA (Karunatillake et al., 2020)), including 25\% reserve cost for development (phase B-D).

References Abilleira et al. (2019) Abstract \#AAS 19-203 - Adeli et al. (2019) 10.1029/2018JE005772 - Agle \& Johnson (2019) https:/www.jpl.nasa.gov/news/news.php?feature=7361 - Baker and Carter (2019a) 10.1016/j.icarus.2018.09.001 - Baker and Carter (2019b) 10.1016/j.icarus.2018.09.009 - Balaram et al. (2018) 10.2514/6.2018-0023 - Bapst et al. (2018) 10.1016/j.icarus.2017.10.004 - Bapst et al. (2019) 10.1029/2018JE005786 - Becerra et al. (2017) 10.1002/2016GL071197 - Beegle et al. (2009) 10.1002/9783527626625.ch8 - Bell et al. (2017) 10.1002/2016EA000219 - Bramall et al. (2005) 10.1029/2005GL024236 - Bramall \& Nathan (2007) http://adsabs.harvard.edu/abs/2007PhDT .......148B - Bramson et al. (2015) 10.1002/2015GL064844 - Bramson et al. (2017) 10.1002/2017JE005357 - Bramson et al. (2019) 10.1029/2018JE005806 - Bramson et al. (2020) submitted whitepaper: Mid-Latitude Ice on Mars: A Science Target for Planetary Climate Histories and an Exploration Target for In Situ Resources, and 7th Mars Polar Sci. Conf. 2020 Abstract \#6015 - Brough et al. (2019) 10.1016/j.epsl.2018.11.031 - Byrne et al. (2009) 10.1126/science.1175307 - Campbell \& Morgan (2018) 10.1002/2017GL075844 - Carsey et al. (2005) 36th LPSC Abstract \#1844 - Christensen et al. (2003) $10.1029 / 2003 J E 002117$ - Clark et al. (2017) 10.1017/S1473550417000313 - Dickson et al. (2012) 10.1016/j.icarus.2012.03.010 - Dundas et al. (2014) 7th Mars Polar Sci. Conf. Abstract \#1375 - Dundas et al. (2018) 10.1126/SCIENCE.AAO1619 - Franz et al. (2014) 10.1016/j.pss.2014.03.005 - Franz et al. (2017) 10.1038/ngeo3002 - Glass et al. (2014) 10.1002/rob.21487 - Good et al. (2015) 10.1126/science.aaa5931 - Grima et al. (2009) $10.1029 / 2008$ GL036326 - Grotzinger et al. (2012) 10.1007/s11214-012-9892-2 - Harish et al. (2020) 10.1029/2020GL089057 - Hecht \& Saunders (2003) Cryoscout: a descent through the Mars polar cap, Abstract \#032292 - Holt et al. (2008) 10.1126/science.1164246 - Hvidberg et al (2012) 10.1016/j.icarus.2012.08.009 - Landis et al. (2016) 10.1002/2016GL068434 - Lefort et al. (2009) 10.1029/2008JE003264 - Livingston et al. (2002) 10.1021/jp014438c - Lorenzo et al. (2019) 50th LPSC Abstract \#3246 - Kadish \& Head (2011) 10.1016/j.icarus.2011.07.014 - Karunatillake et al. (2020) Table STM 0.17632/hnzwyfrzvg.1 - Krasnopolsky (2015) 10.1016/j.icarus.2015.05.021 Mahaffy et al. (2012) 10.1007/s11214-012-9879-z - Mahaffy et al. (2015) 10.1126/science.1260291 - Maki et al. (2012) 10.1007/s11214-012-9882-4 - Markland et al. (2008) 10.1063/1.2925792 - Malin et al. (2017) 10.1002/2016EA000252 - Martin et al. (2015) 10.1007/s12567-015-0091-3 - McKay et al. (2013) 10.1089/ast.2012.0878 - Mesick et al. (2018) 10.1109/NSSMIC.2018.8824376 - Nagihara et al. (2019) 50th LPSC Abstract \#1557 - NAS (2017) 10.17226/24857 - NAS (2018) 10.17226/25186 - NRC (2011) 10.17226/13117 - NASA "Mission: Spacecraft" - NASA (2014) https://science.nasa.gov/about-us/science-strategy/ - Niles \& Michalski (2009) 10.1038/ngeo438 - Ojha et al. (2019) 10.1029/2019GL082294 - Orgel et al. (2019) $10.1029 / 2018 J E 005664$ - Orosei et al. (2018) 10.1126/science.aar7268 - Pathare et al. (2018) 10.1016/j.icarus.2017.09.031 - Petersen et al. (2018) 10.1029/2018GL079759 - Phillips et al. (2011) $10.1126 /$ science.1203091 - Piqueux et al. (2019) 10.1029/2019GL083947 - Plaut et al. (2009) 10.1029/2008GL036379 - Putzig et al. (2018) 10.1016/j.icarus.2017.09.023 - Quinn et al. (2019) Europa Luminescence Microscope in 2019 Astrobio. Sci. Conf. - Ramsdale et al. (2019) 10.1029/2018JE005663 - Retherford et al. (2019) 50th LPSC Abstract \#3141 - Rodriguez-Manfredi et al. (2014) LPSC and https:/mars.nasa.gov/mars2020/mission/instruments/meda/for-scientists/ - Séjourné et al. (2018) 10.1029/2018JE005665 - Sizemore et al. (2015) 10.1016/j.icarus.2014.04.013 - Smith \& Holt (2010) 10.1038/nature09049 - Smith et al. (2013) doi:10.1002/jgre.20142 - Smith et al. (2016) 10.1126/science.aad6968 Stuurman et al. (2016) 10.1002/2016GL070138 - Sori and Bramson (2019) 10.1029/2018GL080985 - Viola et al. (2015) 10.1016/j.icarus.2014.10.032 - Vos et al.. (2019) 10.1016/j.icarus.2019.01.018 - Whitten et al. (2017) 10.1002/2017GL074069 - Zacny et al. (2008) 10.1089/ast.2007.0179 - Zacny et al. (2012) 10.2514/6.2012-5168 Zacny et al. (2019) 9th Int. Conf. on Mars Abstract \#6333 - Zacny et al. (in prep, 2020) Advances in Terrestrial and Extraterrestrial Drilling: Ground, Ice, and Underwater 